\title{
Choroid plexus papilloma of the fourth ventricle in a septuagenarian
}

\author{
Alok A Umredkar, Rajesh Chhabra, Aman Bal ${ }^{1}$, Ashim Das ${ }^{1}$ \\ Departments of Neurosurgery, ${ }^{1}$ Pathology, Postgraduate Institute of Medical Education and Research, Chandigarh, India
}

\section{ABSTRACT}

Choroid plexus papillomas (CPP) are most commonly found in children and rarely in adult. A very few cases are reported in geriatric population. We present a case of 72 -year-old man with posterior fossa lesion operated with provisional diagnosis of metastasis, but turn out to be CPP.

Key words: Choroid plexus, geriatric tumour, ventricular tumours

\section{Introduction}

Choroid plexus papillomas (CPPs) are rare tumors of neuroectodermal origin accounting for less than $1 \%$ and mainly occur in young children with a favorable long-term prognosis. ${ }^{[1]}$ It has been reported that $70 \%$ of the tumor occurs in children and at least $50 \%$ presents before the age of $2 .{ }^{[2]} \mathrm{A}$ very few reports of $\mathrm{CPP}$ in adult are present in the literature. ${ }^{[2-5]}$

\section{Case Report}

A 72-year-old male presented with imbalance while walking for the past 1 year and headache and vomiting for 2 months. The patient did not have any medical history or family history related to brain lesion. His visual acuity was mildly decreased (0.6/0.4), but visual field was within normal limits. Brain MRI showed a hypo- to isointense $55 \times 47 \times 44 \mathrm{~mm}$ sized mass occupying the whole of fourth ventricle with associated cyst and obstructive hydrocephalous on T1-weighted images [Figure 1a], and intermediate signal intensity on T2-weighted images with only slight extent of perilesional edema [Figure 1b].

\begin{tabular}{|l|l|}
\hline \multicolumn{2}{|c|}{ Access this article online } \\
\hline Quick Response Code: & Website: \\
\hline & www.ruralneuropractice.com \\
\cline { 2 - 3 } & \\
\hline & \\
\hline
\end{tabular}

This tumor was intensely enhanced on contrast study [Figures $1 \mathrm{c}$ and $\mathrm{d}$ ]. Considering the age of the patient and MRI characteristics, a provisional diagnosis of metastasis was made. Ventriculo-peritoneal shunting was done for hydrocephalous. After doing the workup for primary, he underwent sub-occipital craniotomy, and gross total excision. The tumor was greyish, soft, suckable, and very vascular; gross total excision was done. Histopathology revealed a papillary tumor with mild nuclear pleomorphism and hyperchromasia

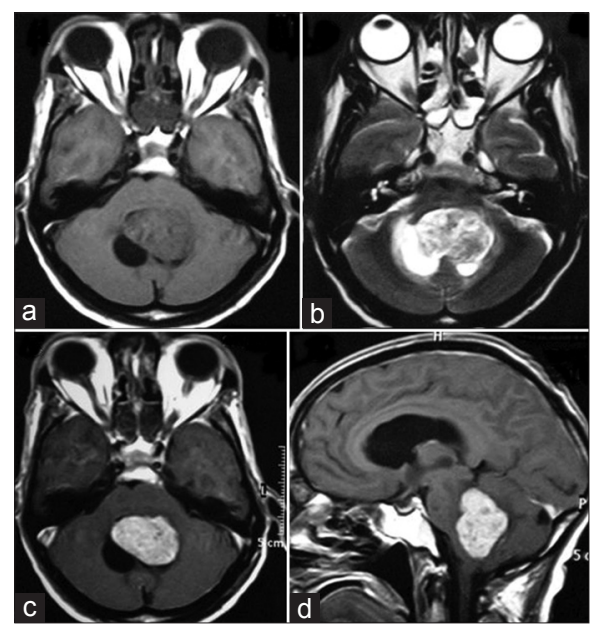

Figure 1: (a) Brain MRI showing a hypo- to isointense $55 \times 47 \times 44 \mathrm{~mm}$ sized mass occupying the whole of fourth ventricle with associated cyst on T1-weighted image. (b) MRI scan showing same tumor with hypo- to intermediate signal intensity on T2-weighted images with only slight perilesional edema. (c) Axial section CEMRI scan showing same tumor with intense contrast enhancement. (d) Sagittal section CEMRI scan showing same tumor occupying whole fourth ventricle 
without nuclear atypia, mitosis, and necrosis, suggesting diagnosis of $\mathrm{CPP}$ [Figures 2a and b]. Postoperative period was uneventful. Patient did not receive radiotherapy or chemotherapy. At 1-year follow-up, patient was doing well, and contrast-enhanced MRI showed no recurrence of tumor [Figures $2 \mathrm{c}$ and d].

CPPs are accounting for $1-5 \%$ of pediatric brain tumors and $4-12 \%$ among patients younger than 1 year old. ${ }^{[2]}$ In childhood, CPPs are usually found in the lateral ventricles and less commonly in the posterior fossa. On the contrary, in adults, the majority of these tumors are found in the fourth ventricle and its lateral recesses. ${ }^{[2]}$ Very few reports of adult cases are found in the literature and that in seventh decade, still not reported.

CPPs are classified as World Health Organization Grade I tumors. Because of the benign nature of these tumors, total surgical resection is typically thought to be curative. The malignant form, CPC is classified as a World Health Organization Grade III tumor and is characterized by anaplasia, mitosis, nuclear pleomorphism, necrosis, and invasion. ${ }^{[5]}$ The atypical CPP is a newly introduced entity as an intermediate grade in the $2007 \mathrm{WHO}$ central nervous system (CNS) tumor classification. This tumor is mainly distinguished from the CPP by increased mitotic activity, 2 or more mitoses per 10 high power fields (HPF) while usually greater than 5 per $10 \mathrm{HPF}$ in CPC. ${ }^{[2]}$ The majority of choroid plexus tumors are well-differentiated benign neoplasm. Macroscopically, CPPs appear as circumscribed cauliflower-like masses that may attach to the wall of ventricle but are usually well marginated from normal

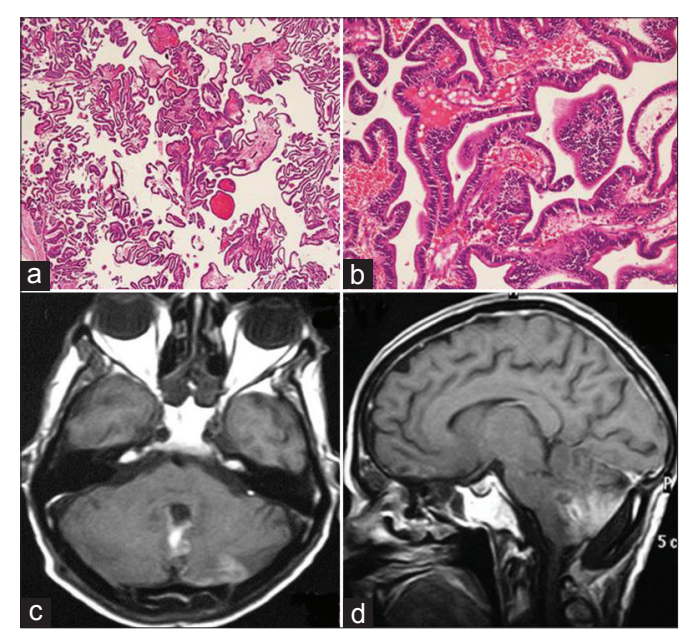

Figure 2: (a) Photomicrograph showing papillary tumor with columnar epithelial cells resting on distinct fibrovascular core (Hematoxylin and eosin, $\times 40$ ). (b) Photomicrograph showing columnar epithelium without much cytological atypia, resting on continuous basement membrane (Hematoxylin and eosin, $\times 200$ ) (c) axial section CEMRI scan showing no recurrence after 1 year of surgery. (d) Sagittal section CEMRI scan showing no recurrence after 1 year of surgery brain structures. Microscopic findings of CPPs typically appear as a single layer of cuboidal epithelial cells surrounding a fibrovascular stalk, arranged in a papillary configuration with finger-like projections. The histological features of CPC characterized by signs of malignancy includes brisk mitoses, nuclear pleomorphism, raised cellular density, obscurity of the papillary growth pattern, and cell necrosis. ${ }^{[2]} \mathrm{CPPs}$ are typically considered benign lesions, but histology is not always predictive of their behavior. These tumors can metastasize anywhere along the neuraxis and may be intraventricular, subarachnoid, or intraparenchymal. ${ }^{[3,4]}$ Genetic transition including p53 mutation (location is at locus p53, 191170.0036) and hSFN5/IN/1 somatic mutations of chromosome 22 were reported in CPP, atypical CPP, and choroid plexus carcinomas in the same family. CPPs in siblings or identical twins were reported twice previously. ${ }^{[1]}$

Hydrocephalus is usually an associated feature of CPPs. Causes includes overproduction of CSF, inflammation, and obstruction of CSF pathways. ${ }^{[1]}$ Extraventricular growth of choroid plexus tumors is found in three situations: direct extension of the primary tumour, seeding along cerebrospinal fluid pathways, and development of a primary tumor from the small choroid tuft that normally project outside the foramen of Luschka. ${ }^{[5]}$ In our case, the tumor was arising from the fourth ventricular wall and occupying the whole ventricular cavity causing obstructive hydrocephalous.

The CT characteristics of CPT are well characterized. On nonenhanced studies, the tumor appears as a smooth or lobulated mass, hyperdense in relation to surrounding brain parenchyma. With intravenous contrast, there is marked, homogeneous enhancement. With MRI these tumors showed an isointensity in T1-weighted images and iso-hypointensity in T2-weighted images, with marked enhancement after gadolinium. ${ }^{[3,4]}$ These may have intratumoral signal voids that correspond to a rich vascular supply or low-intensity areas that correspond to calcifications. Angiographic signs of CPPs may include the presence of many small spiral arteries; a meningiomatype blush with early tumoral circulation and persistent enhancement into the venous phase; displacement of vessels such as the internal cerebral veins; and evidence of ventricular dilatation. The differential diagnoses of posterior fossa CPPs are medulloblastomas, typical teraoid/rhabdoid tumor (AR/AT), ependymomas, and metastasis tumor. ${ }^{[4]}$

The standard treatment for a CPP is complete microsurgical resection, typically thought to be curative. ${ }^{[1]}$ In our patient, a midline suboccipital craniectomy was done. The cisterna magna was opened and the tonsils 
were separated. The tumor was encountered and its surface coagulated. It was removed after shrinking it by coagulation and irrigation. Tacconi et al. reported a good outcome if the resection was complete with a recurrence rate of less than $10 \%{ }^{[1]}$ Radiation therapy has been used in cases of CPP with subtotal resection and stereotactic radiosurgery has been reported when a patient refuses surgery for treatment of a small CPP. Chemotherapy in CPP is limited and there is no consensus on its impact in the management of CPP. Valencak et al. reported the therapeutic efficacy of CCNU in recurrent CPP and extensive spinal seeding. There are two case reports suggesting that CCNU may be effective in CPP. ${ }^{[5]}$

We experienced a rare case of a septuagenarian patient with fourth ventricular CPP and achieved successful surgical result after complete excision. So, there seems to be a chance of cure and low risk of recurrence for CPPs if the resection is complete. Role of radiotherapy in CPP treatment is still controversial and it is better to reserve this alternative just for recurrent and residual lesions with a risk of high morbidity and mortality. Follow-up is therefore the best choice to handle these patients.

\section{References}

1. Okay O, Dağlioğlu E, Yakicier C, Uren D, Dalgiç A, Ergüngör F. Choroid plexus papillomas in two siblings: Case report. Turk Neurosurg 2009;19:281-4.

2. Lee SH, Park BJ, Kim EJ, Lim YJ. Atypical choroid plexus papilloma in an adult. J Korean Neurosurg Soc 2009;46:74-6.

3. Jinhu Y, Jianping D, Jun M, Hui S, Yepeng F. Metastasis of a histologically benign choroid plexus papilloma: Case report and review of the literature. J Neurooncol 2007;83:47-52.

4. McCall T, Binning M, Blumenthal DT, Jensen RL. Variations of disseminated choroid plexus papilloma: 2 case reports and a review of the literature. Surg Neurol 2006;66:62-8.

5. Ahn SS, Cho YD. Spinal drop metastasis from a posterior fossa choroid plexus papilloma. J Korean Neurosurg Soc 2007;42:475-7.

How to cite this article: Umredkar AA, Chhabra R, Bal A, Das A. Choroid plexus papilloma of the fourth ventricle in a septuagenarian. $J$ Neurosci Rural Pract 2012;3:402-4.

Source of Support: Nil. Conflict of Interest: None declared. 